\title{
LA EQUIDAD DE GÉNERO EN LAS DECISIONES DE CONSUMO Y AHORRO DE LAS FAMILIAS, PARROQUIA EL RETIRO, PROVINCIA DE EL ORO
}

\section{GENDER EQUITY IN THE CONSUMPTION AND SAVING DECISIONS OF FAMILIES OF THE RETIRO PARISH, PROVINCE OF EL ORO}

Eco. Lady Andrea León Serrano, Mg

llady@utmachala.edu.ec

Universidad Técnica de Machala (UTMACH), Ecuador

\section{RESUMEN}

El presente trabajo de investigación tiene como objetivo principal conocer la influencia de la equidad de género en las decisiones de consumo y ahorro de las familias parroquia El Retiro ubicado en el cantón Machala, Provincia de El Oro. El desarrollo se establece en base al análisis de la equidad de género de las familias del sector y el impacto en los niveles de gasto y ahorro de las economías domésticas, contrastando mediante el método muestreo probabilístico con aplicación de instrumentos: una entrevista al jefe de la junta parroquial y veinte encuestas a familias con una media de cuatro miembros, hogares seleccionados aleatoriamente con ingresos promedios mensuales de $\$ 567,00$. Los resultados de la recolección de datos destacaron al hombre como jefe de hogar e influyente en las determinantes de los rubros básicos por ser el único perceptor de ingresos, la mujer en su rol de cuidado de hogar y la existencia de un porcentaje considerable de mujeres dedicadas a las labores de campo, destacan a la economía familiar con un nivel de ahorro debajo del 10\% supeditado por la renta. Las decisiones económicas de los miembros se acuerdan mutuamente, corroborando los resultados con las bases teóricas de consumo y ahorro.

Palabras clave: Equidad, Género, Consumo, Ahorro, Familias

\section{ABSTRACT}

The present work of research has as objective main know the influence of the equity of gender in them decisions of consumption and saving of them families parish El Retiro removal located in the canton Machala, province of El Oro.

Development is set on the basis of the analysis of the families of the sector and the impact gender equity to levels of spending and saving of domestic economies, contrasting with application of instruments probabilistic sampling method: an interview with the head of the vestry and twenty surveys to families with an average of four members, households randomly selected income monthly averages of \$567,00. The results of data collection stressed the man as head of household and influential on the determinants of the headings Basic for being the only beneficiary income, women in their role of home care and the existence of a considerable percentage of women engaged in field work, include the family economy with a level of savings under $10 \%$ subordinated by the income. The economic decisions of the members agree mutually, corroborating the results with theoretical bases of consumption and savings.

Key words: Equity, Gender, Consumption, Savings, Families

Recibido: 9 de octubre de 2016

Aceptado: 25 de enero de 2017

Publicado: 30 de junio de 2017 


\section{INTRODUCCIÓN}

A comienzos de siglo, líderes de varios países se reunieron en la Naciones Unidas para suscribir los Objetivos del Desarrollo del Mileno (ODM) tomando como fundamento la igualdad de género entre hombres y mujeres. De la socialización de género se aprende la importancia de los valores, creencias, emociones o los comportamientos socialmente aceptables para hombres y mujeres (1). Los cambios generados por las crisis económicas, especialmente desde el 2008, han afectado varias naciones especialmente europeos, en la vulnerabilidad de las mujeres, sin embargo América Latina, a pesar de haber mejorado levemente la situación, existe el aumento de los casos de autonomía económica y roles sociales por parte de la mujer (2).

La equidad de género implica decisiones centradas en relación de poder entre ambos sexos, en los obstáculos que enfrenta el sector femenino en la búsqueda de su independencia en los sectores sociales. La igualdad de mujeres $y$ hombres es un factor de derecho de trato y oportunidades igualitarias así como la no discriminación e integración de ambos sexos en el desarrollo social económico (3). La discriminación de género tiene como prácticas sociales reacomodar los avances normativos al respecto (4). Estos avances recaen en los cambios sociales, en los valores individuales, en el impacto económico y el rol desempeñado por las cabezas de hogar en las decisiones compra $y$ ahorro de las familias.

El consumismo se lo define como un atributo de la sociedad conformada por individuos cuya capacidad de querer, desear o anhelar forma parte del principal motor de rotación de la economía, desplazando al trabajo de su principal actividad generadora de la sociedad (5). El consumo es uno de los grandes componentes de la demanda agregada por la relación con el ahorro e inversión, la distribución de gastos de las familias se determina por las desigualdades continuas surgidas (6).

A lo largo de las décadas el consumo familiar ha ido evolucionando, las repetidas crisis globales han repercutido sobre el nivel de consumo, las transiciones a la era industrial pasaron por momentos sociales ajustados, en el que Japón marcó el cambio seguido por los países "dragones asiáticos". Con la llegada de la tecnología, se generó el cambio sobre las preferencias en el consumo y la diversificación en los productos, marcando que los bienes no quedan obsoletos por la pérdida de su valor funcional sino porque dejan de ser atractivos (7).

La dinámica de la toma de decisiones familiares son por los hombres y la poca influencia de las mujeres, este menor porcentaje de sector femenino decide sobre lo referente a la salud, compras domésticas gastos diarios del hogar, de tal manera que al ganar menos dinero, tienen menos bienes, esto da a conocer la distribución del poder de negociación en los hogares (8). Es así que, la satisfacción de necesidades mejora el bienestar económico social de los hogares acompañados de la equidad de género e igualdad de decisiones (9).

Ecuador ha pasado diferentes fluctuaciones en el ahorro interno, manteniéndose en niveles por debajo de lo necesario para lograr un crecimiento económico estable. Desde la última década, el ahorro decreció $15.7 \%$ en 1985 a $7.6 \%$ en 1986 , con un ascenso posterior de $11.8 \%$ en 1991 (10). En el censo realizado en las principales provincias del Ecuador 2011-2012, determinaron las características del jefe de hogar, con un resultado de $76.2 \%$ hombres y el $23.8 \%$ mujeres; el $39.6 \%$ son jefes entre 25 y 44 años, con promedio de 3,9 miembros en el hogar, el ingreso promedio mensual nacional para el área urbana llegó a USD 1.046,00, con un 76\% destino al gasto de consumo de los hogares (11); esta referencia marca la influencia de mayor consumo y menor ahorro de la familia ecuatoriana.

Con esta percepción, el cantón Machala perteneciente a la provincia de El Oro, goza de ocho parroquias urbanas, que dinamizan la economía por su sector comercial productivo, así mismo parte del movimiento productivo de la economía local se fundamenta en las empresas familiares por los ingresos diarios percibidos 0 por la relación de dependencia en diferentes áreas productivas; las economías domésticas destinan sus gastos a los principales rubros básicos, con una inclinación mayoritaria al consumo y en menor porcentaje al ahorro, que repercute en la falta de un colchón de ahorro. Las economías consumistas gastan más de lo que ganan, reduciendo el ahorro acumulado y aumentando el endeudamiento familiar con futuros problemas de déficit en el presupuesto personal (12). La base central del tema de investigación es conocer la influencia de la equidad de género en las decisiones de consumo y ahorro de las familias pertenecientes a la parroquia rural El Retiro, considerando el ingreso promedio mensual y el poder de compra familiar desde la perspectiva Keynesiana. 


\section{La propensión marginal al consumo y al ahorro en la teoría de Keynesiana}

La obra de John Maynard Keynes, Teoría general, publicada en 1936, explica la función de consumo como el fundamento para explicar las fluctuaciones económicas y los análisis macroeconómicos derivados de la misma. En base a varios análisis sobre las conjeturas en la función de consumo se basa en la introspección y observación del entorno (13). Keynes seguido de su observación en el comportamiento social europeo durante la crisis de los 30 , la propensión marginal al consumo y al ahorro fueron parte de las propuestas para explicar las recesiones económicas con la aplicación de políticas que aumenten el flujo monetario $y$ gasto gubernamental. La teoría keynesiana de consumo, ahorro e inversión comprende los determinantes que recaen sobre el ingreso disponible de las familias distribuidas en el consumo y ahorro (14).

La propensión marginal al consumo definiéndola como la cantidad consumida de una moneda adicional de la renta, por regla general la gente aumenta su consumo cuando aumenta la renta, pero en cantidades menores (13), otros pensadores (14) destacan a la propensión marginal al consumo (PMC) como la proporción extra que se destina al gasto de consumo por cada moneda extra. A diferencia de la propensión marginal al ahorro (PMS) es la variación en el ahorro total, resultante de un cambio en el ingreso (15).

El ahorro y la inversión son dos actos independientes y realizados por individuos diferentes, el mercado no garantiza la igualdad entre las variables, sin embargo el ahorro depende del ingreso es decir de la capacidad de ahorro de los individuos o las familias, sin negar que las tasas de interés influyen en las partes ahorradas. Cuando el ingreso es mayor la capacidad de ahorro aumentará de los individuos y familias, generando un ahorro general en la economía (16).

Keynes, afirmó que las personas están dispuestas a aumentar su consumo si se incrementa su ingreso pero no en la misma proporción en que lo hace este último, esta hipótesis es el supuesto formal de la función de consumo que sustenta el modelo. Las funciones consumo y ahorro en forma general $C^{*}$ y $A^{*}$ son las propensiones marginales al consumo $y$ al ahorro respectivamente. Ante estas posibilidades, las dos son positivas pero menores a uno, por lo tanto la relación entre el consumo y el ingreso disponible es la PMC mientras que la relación ahorro e ingreso disponible es la PMS, es decir la propensión marginal al consumo debería ser superior a la propensión marginal al ahorro; determinando que la función keynesiana del ahorro tiene una relación lineal y directa con el ingreso disponible (10).

Otro aspecto son los factores en el largo plazo de las tasas de interés en la teoría de bienes sobre la importancia de la propensión a ahorrar en las economías y las oportunidades existentes al momento de invertir (17). El funcionamiento del sistema económico se compensa por el rendimiento de las economías, es decir los países deben contar con un considerable nivel de ahorro interno proveniente del producto interno bruto que no se destinará al gasto público sino para el crecimiento y desarrollo socioeconómico de los estados (10).

América Latina se ha caracterizado por registrar tasas de ahorro bajas, con el objeto de alcanzar un crecimiento económico sostenido, a través de la tasa ideal de ahorro interno, para esto es necesario los factores que ayudan a encontrar una inversión doméstica adecuada para consolidar altas tasas de crecimiento. Ecuador desde la evolución irregular del periodo 1965 1995 sobre la tasa de ahorro nacional, logró altos niveles de ahorro nacional bruto desde el boom petrolero. En 1993, los hogares y sector público enfrentaron un decrecimiento en los ahorros, hasta llegar al $14.95 \%$ en 1995 , los elementos que llevaron a esta baja fue causado por el aumento de la tasa de consumo final desde hace cinco años atrás, en especial por el consumo de las Administraciones Públicas (18).

En efecto, parte de las causas para la reducción de la tasa de ahorro nacional bruto caso ecuatoriano, serían el estancamiento en el ritmo de crecimiento económico, una menor disciplina fiscal, aumento de la estructura de la población menor restricción del crédito del consumo y un menor ahorro frente a tasas de interés altas (18)..

El ingreso disponible per cápita a precios constantes tendría un efecto positivo especialmente en el gasto a la educación, ya que al aumentar el nivel de educación se incremente el nivel de los ingresos y la capacidad de ahorro en las familias (18).

\section{MATERIALES Y MÉTODOS}

El esquema utilizado está basado en el método cuantitativo probabilístico, para identificar el nivel 
de consumo y ahorro en las familias de la parroquia El Retiro del cantón Machala determinado por la equidad de género. Los instrumentos de investigación aplicados fueron a través de entrevista y encuestas. Las encuestas (19) se utilizan para estudiar poblaciones mediante el uso de muestras valorativas para explicar las variables de estudio y su comportamiento.

El cantón Machala, Capital Bananera del Mundo, perteneciente a la Provincia de El Oro, cuenta con 217.696 habitantes (20). Formada por siete parroquias urbanas y una rural El Retiro. Las ramas de actividad de la población ocupada en el cantón Machala por: el comercio al por mayor y menor en $28.1 \%$ la agricultura, ganadería, silvicultura y pesca en $14.8 \%$, industria manufacturas $7.9 \%$ entre otros, el $42.6 \%$ corresponde a la PEA del total de El Oro (21). Según la encuesta nacional 2011-2012, el 81.6\% son jefes de hogar masculinos en el sector rural (11).

EI GAD Parroquial El Retiro (22), la parroquia está constituido por 4.367 habitantes, la localidad está estructurada por once sitios. La superficie llega a $127 \mathrm{Km} 2$, ubicada a $17.5 \mathrm{Km}$ de distancia a la ciudad de Machala, la actividad principal es la agrícola en el cultivo y exportación de banano, pesca y comercio (construcción).

Para la investigación se aplicó la entrevista al representante de la Junta parroquial El Retiro y para las encuestas la muestra de la población universo se consideró a veinte familias aleatoriamente, (22) la mayor parte de la población está empleada como jornalera en las haciendas bananeras aledañas, principal actividad comercial de la parroquia e incide en la economía local la variación de los precios de la caja de banano. Las familias seleccionadas para la investigación forman el cien por ciento de la muestra objeto de estudio.

En la primera fase se aplica la entrevista al representante de la Junta parroquial EI Retiro con el objeto de conocer el aporte económico de las familias en las actividades productivas de la localidad y la incidencia del rol del jefe (a) de hogar en las decisiones domésticas.

La segunda fase tuvo por objeto identificar la influencia de la equidad de género en las decisiones económicas del hogar supeditadas al gasto y ahorro. Se triangularon los resultados de la entrevista y las encuestas con las bases teóricas en base a la función de consumo, ahorro y renta percibida. Ésta última etapa permitió corroborar la información de los instrumentos utilizados para establecer si se cumple la teoría Keynes incluyendo como factor de estudio la participación equitativa de los miembros del hogar ante la presencia de un solo perceptor de ingresos como eje de calidad de la vida doméstica.

\section{RESULTADOS}

De acuerdo a la entrevista efectuada al representante de la Junta parroquial, se determinó que El Retiro tiene varias actividades económicas, de la cual las familias participan activamente en especial en el sector banano desempeñando funciones de jornaleros y más labores de campo, el hombre se desempeña como jefe de hogar y como único perceptor de ingresos, la mujer participa en el cuidado del hogar, la proporción de mujeres que laboran son por situaciones personales entre una de ellas ser madres solteras por la necesidad de generar ingresos para el hogar, actualmente la inclusión del hombre y mujer en labores de trabajo productivo es equitativo, dando oportunidades a ambos géneros, ocasionando cambios sociales domésticos, aclarando que es una localidad que depende de los precios comerciales de la fruta de banano de exportación.

En el desarrollo de la segunda parte de las encuestas aplicadas, se tabularon los datos acerca de la influencia de la equidad de género en las decisiones de consumo y ahorro de las familias parroquia El Retiro, presentando el análisis e interpretación de los datos obtenidos de las veinte familias, mediante tablas y gráficos explicativos e interpretación teórica de los mismos.

Tabla 1. Edad

\begin{tabular}{|c|c|}
\hline Rango de edad & $\begin{array}{c}\mathbf{N}^{\circ} \\
\text { Observaciones }\end{array}$ \\
\hline $18-32$ & 1 \\
\hline $33-44$ & 5 \\
\hline $45-55$ & 8 \\
\hline $56-67$ & 4 \\
\hline 68 en adelante & 2 \\
\hline Total & 20 \\
\hline
\end{tabular}

Los datos de la Tabla 1 detallan la edad promedio de los encuestados entre un rango de 45 - 55 años en su mayoría, seguidos del rango 33 - 44 y 56 - 67 años como segunda y tercera categoría respectivamente frente a un bajo porcentaje de familias entre las edades de 18 32 años de los valores obtenidos, se determina la existencia de hogares con promedio de edad mayor a 45 años. 


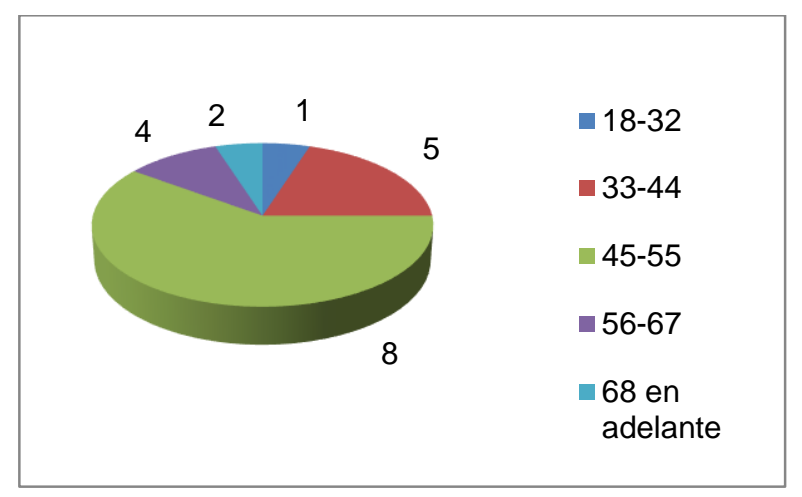

Figura 1. Edad promedio en los hogares encuestados

Tabla 2. Sexo

\begin{tabular}{|c|c|}
\hline Sexo & $\mathbf{N}^{\circ}$ Observaciones \\
\hline Masculino & 14 \\
\hline Femenino & 6 \\
\hline Total & 20 \\
\hline
\end{tabular}
porcentaje por el género masculino, además de su rol en la ocupación de la economía de la localidad, seguido de las mujeres con menor porcentaje. Tabla 2.

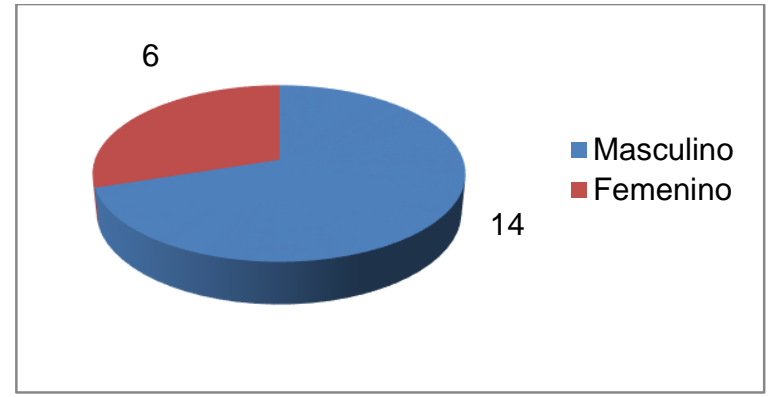

Figura 2. Numero de género encuestados

Tabla 3. ¿Cuál de los miembros de la familia aporta económicamente en el hogar?

\begin{tabular}{|c|c|}
\hline Miembros de la Familia & $\begin{array}{c}\mathbf{N}^{\circ} \\
\text { Observaciones }\end{array}$ \\
\hline Hombre & 12 \\
\hline Mujer & 5 \\
\hline Los dos & 3 \\
\hline Hijos & 0 \\
\hline Total & 20 \\
\hline
\end{tabular}

Otro factor con incidencia es el número de miembros que aportan económicamente en el hogar, el resultado apunta al hombre como el mayor perceptor de ingresos (12), seguido de 5 encuestas inclinadas a las mujeres y en la opción de los dos miembros aportan a la renta familiar, es considerable este factor en la marcada actuación del hombre en el hogar. Tabla 3.

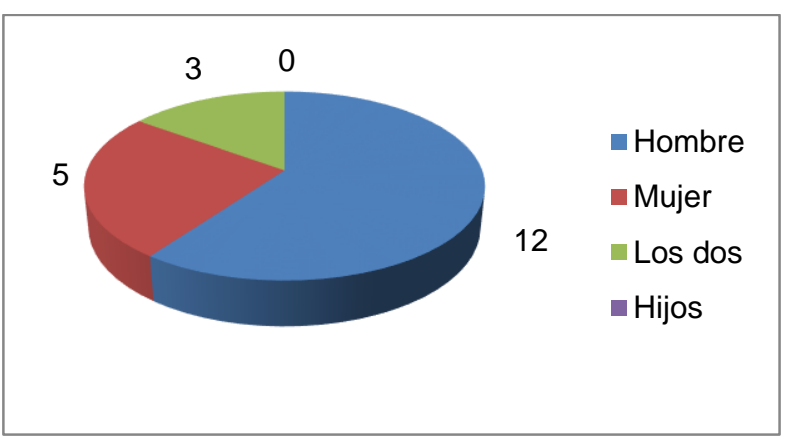

Figura 3. Número de miembros aportan en la renta del hogar

Tabla 4. ¿Cuántas personas integran su hogar?

\begin{tabular}{|c|c|}
\hline Personas & $\mathbf{N}^{\circ}$ Observaciones \\
\hline 1 a 4 & 17 \\
\hline 5 a 7 & 3 \\
\hline 8 o más & 0 \\
\hline Total & 20 \\
\hline
\end{tabular}

Al analizar el número de integrantes en los hogares, existe un promedio de $1-4$ miembros por familia, la incidencia es fuerte por la distribución de los recursos en el hogar, otro grupo minúsculo supera los 4 miembros de la primera categoría, sin embargo forma parte de la población económicamente activa de la parroquia. Tabla 4.

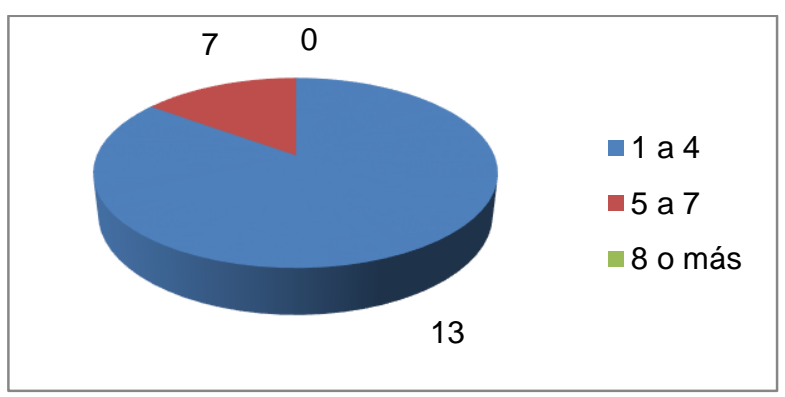

Figura 4. Número de miembros que integran la familia

Tabla 5. De la familia que usted pertenece ¿Quién se desempeña como Jefe(a) de hogar?

\begin{tabular}{|c|c|}
\hline Jefe (a) de hogar & $\begin{array}{c}\mathbf{N}^{\circ} \\
\text { Observaciones }\end{array}$ \\
\hline Hombre & 15 \\
\hline Mujer & 5 \\
\hline Los dos & 0 \\
\hline Total & 20 \\
\hline
\end{tabular}

El resultado concreta al hombre como jefe de hogar en la mayoría de las familias visitadas y un menor valor ocupa la mujer. El género masculino 
se ha destacado por su rol de llevar la renta al hogar y la protección de los miembros que tenga a su cuidado, sin descartar la valiosa participación de la mujer en esta función por situaciones personales. Tabla 5.

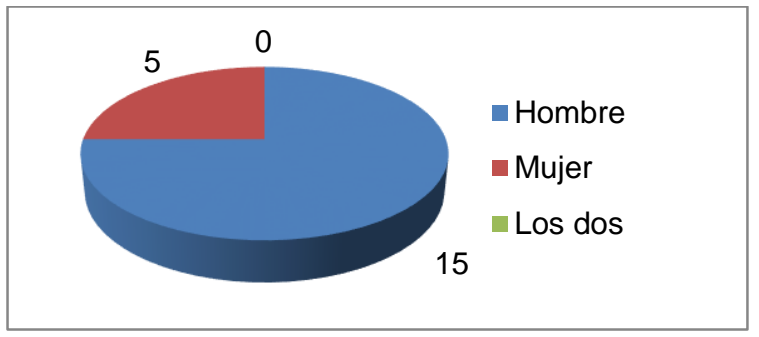

Figura 5. Jefe (a) de hogar

Tabla 6. ¿Cómo familia existe igualdad en las decisiones del hogar?

\begin{tabular}{|c|c|}
\hline Comportamiento & $\begin{array}{c}\mathbf{N}^{\circ} \\
\text { Observaciones }\end{array}$ \\
\hline Siempre & 13 \\
\hline A menudo & 7 \\
\hline Nunca & 0 \\
\hline Total & 20 \\
\hline
\end{tabular}

El análisis de las encuestas demuestran que de las 20 familias, 13 de ellas toman decisiones en igualdad de oportunidades, los consensos son por el hombre y la mujer, un número menor manifestaron que no es habitual establecer consensos sin embargo se respeta el criterio de la pareja. Tabla 6.

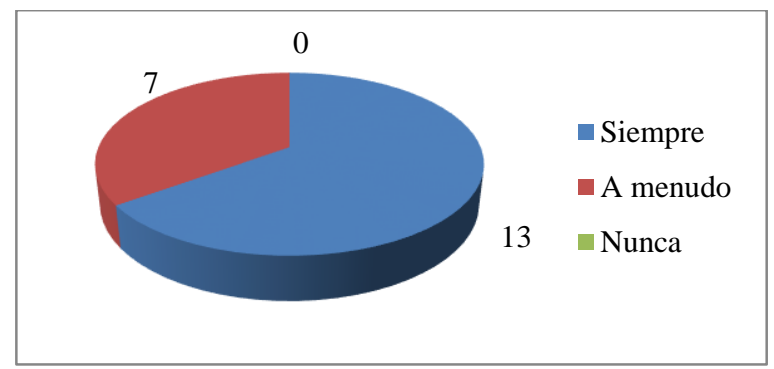

Figura 6. Igualdad de decisiones en el hogar

Tabla 7. Escoja los aspectos, por lo que la mujer o el hombre permanecen en el hogar

\begin{tabular}{|c|c|}
\hline Observaciones & $\begin{array}{c}\mathbf{N}^{\circ} \\
\text { Observaciones }\end{array}$ \\
\hline Cuida del hogar & 10 \\
\hline Negocio & 5 \\
\hline $\begin{array}{c}\text { Escasas oportunidades de } \\
\text { trabajo }\end{array}$ & 3 \\
\hline No es necesario & 2 \\
\hline Total & 20 \\
\hline
\end{tabular}

Referente a la Tabla 7 . Un número de observaciones altas permiten analizar que la pareja permanece en el hogar por motivo de cuidado de la misma, es decir no todos los miembros del hogar principales trabajan, por lo que dependen únicamente del jefe (a) de hogar activo, la diferencia atiende del negocio, otros encuestados mencionaron por las escasas oportunidades de trabajo y un bajo resultado considera no necesario que la pareja labore.

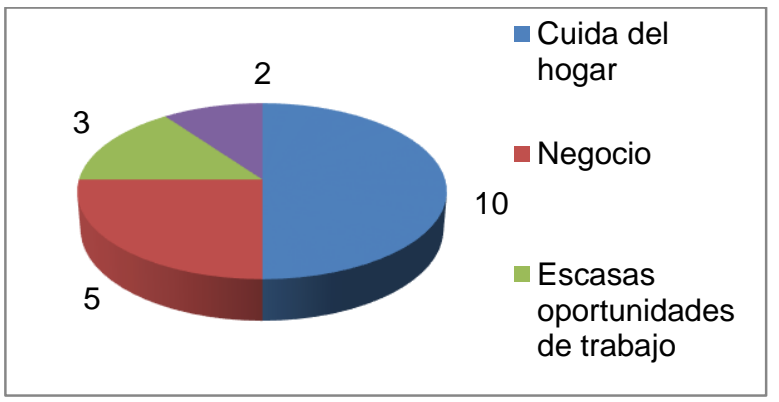

Figura 7. Aspectos que la mujer o el hombre permanecen en el hogar

Tabla 8. ¿Cuánto es el ingreso promedio mensual que percibe la familia?

\begin{tabular}{|c|c|}
\hline Ingreso mensual & $\begin{array}{c}\mathbf{N}^{\circ} \\
\text { Observaciones }\end{array}$ \\
\hline Menos de $\$ 366,00$ & 0 \\
\hline De $\$ 367,00$ a $\$ 466,00$ & 1 \\
\hline De $\$ 467,00$ a $\$ 566,00$ & 8 \\
\hline Más de $\$ 567,00$ & 11 \\
\hline Total & 20 \\
\hline
\end{tabular}

El ingreso mensual promedio percibido por las familias se plantea de más de $\$ 567,00$ influido por el comercio, labores de campo, construcción y pesca; otros hogares su renta tiene un estándar de $\$ 467,00$ a $\$ 566,00$ y solo una familia manifestó percibir ingresos menos de $\$ 466,00$. El nivel de renta depende de la actividad comercial propia del sector, causando serias crisis económicas cuando caen los precios comerciales que afectan al nivel de vida de la parroquia.

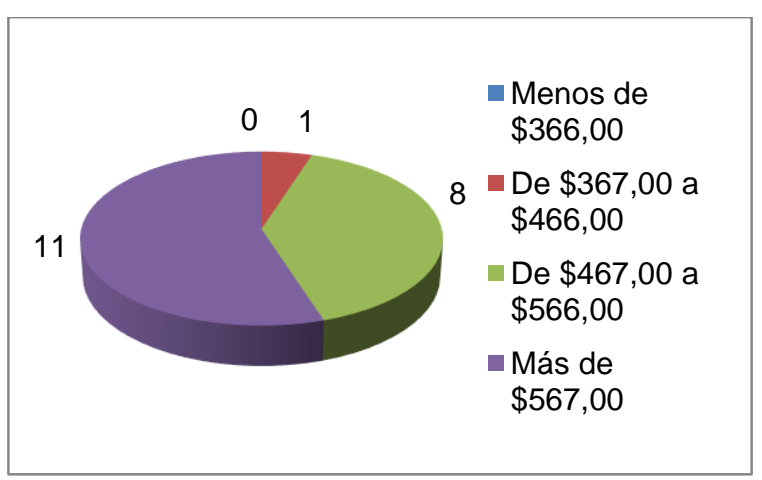

Figura 8. Ingreso promedio mensual de la familia 
Tabla 9. En porcentaje, ¿Cuál es el gasto promedio mensual de los siguientes rubros?

\begin{tabular}{|c|c|}
\hline Rubros gasto consumo & Porcentaje \\
\hline Alimentación & 59 \\
\hline Vestimenta & 7 \\
\hline Educación & 8 \\
\hline Salud & 12 \\
\hline Servicios básicos & 14 \\
\hline Total & 100 \\
\hline
\end{tabular}

Los gastos de consumo por prioridades están orientados como rubro principal de la renta en la alimentación, otro factor de gasto es el pago de los servicios básicos luz, agua, teléfono, internet; la salud consta como tercera categoría, luego se menciona la educación y la vestimenta como último destino. Los resultados priorizan la necesidad de mantener a la familia con alimentos ante las eventualidades de inestabilidad de precios.

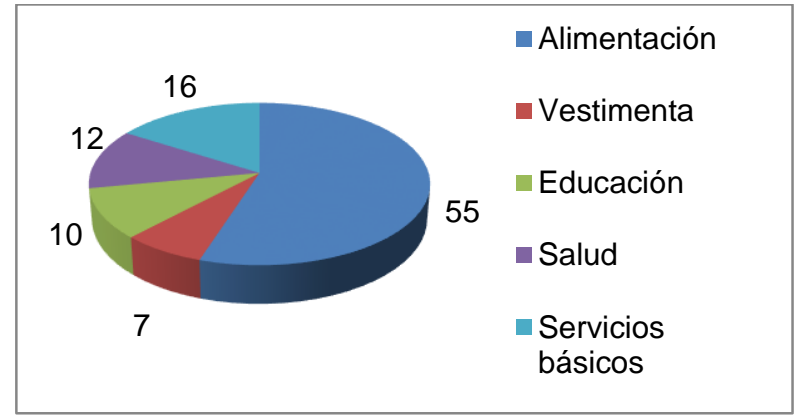

Figura 9. Gastos de consumo familiar mensuales

Tabla 10. ¿Qué porcentaje de los ingresos usted destina al ahorro?

\begin{tabular}{|c|c|}
\hline Ahorro & Porcentaje \\
\hline $0 \%$ & 0 \\
\hline $1 \%-10 \%$ & 17 \\
\hline $10 \%-20 \%$ & 3 \\
\hline $20 \%-30 \%$ & 0 \\
\hline $30 \%$ o más & 0 \\
\hline Total & 20 \\
\hline
\end{tabular}

La estabilidad económica de las familias depende de las actividades comerciales de la localidad, a pesar de los factores el destino del ahorro varía de 1 a $10 \%$, sin embargo este porcentaje de la renta se deteriora o llega a un $0 \%$ de ahorro cuando baja la exportación de banano y camarón, debido que la mayoría de las familias trabajan en fincas. Tabla 10.

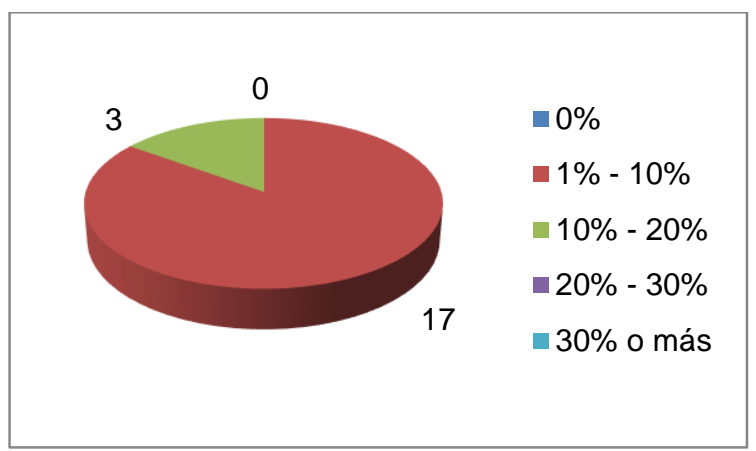

Figura 10. Porcentaje de ahorro familiar

Tabla 11. ¿Qué aspectos considera influyente en su decisión al momento de ahorrar?

\begin{tabular}{|c|c|}
\hline Factores & $\begin{array}{c}\mathbf{N}^{\circ} \\
\text { Observaciones }\end{array}$ \\
\hline Situación económica del país & 0 \\
\hline Compra de bienes & 0 \\
\hline Educación de hijos & 6 \\
\hline Imprevistos & 4 \\
\hline Viajes & 0 \\
\hline Enfermedades & 10 \\
\hline Total & 20 \\
\hline
\end{tabular}

Cada factor es esencial para el destino del ahorro, los encuestados manifestaron que parte de sus ahorros son por motivos de salud para atención de enfermedades de la familia; luego sigue para educación de hijos y por último por los imprevistos ocasionados por pagos 0 eventualidades del hogar. Tabla 11.

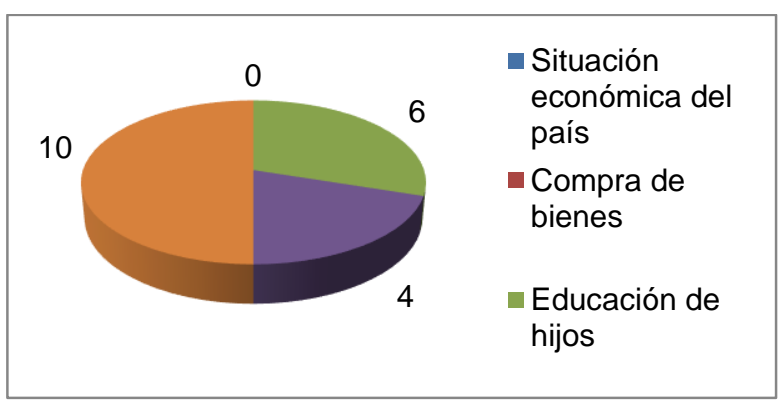

Figura 11. Decisión de ahorro familiar

Tabla 12. ¿Considera que la variación de los precios en bienes y servicios que usted adquiere, influye sobre su nivel de consumo?

\begin{tabular}{|c|c|}
\hline Comportamiento & $\begin{array}{c}\mathbf{N}^{\circ} \\
\text { Observaciones }\end{array}$ \\
\hline Siempre & 20 \\
\hline A menudo & 0 \\
\hline Nunca & 0 \\
\hline Total & 20 \\
\hline
\end{tabular}


Los factores externos desempeñan un factor importante en el ahorro y consumo del hogar, la variación de precios de los bienes y servicios, en especial de los víveres de la canasta básica, siempre afectarán, un aumento de precios propicia a comprar menos y una disminución de los mismos provoca mayor adquisición de víveres afectando al comportamiento del consumidor. Tabla 12.

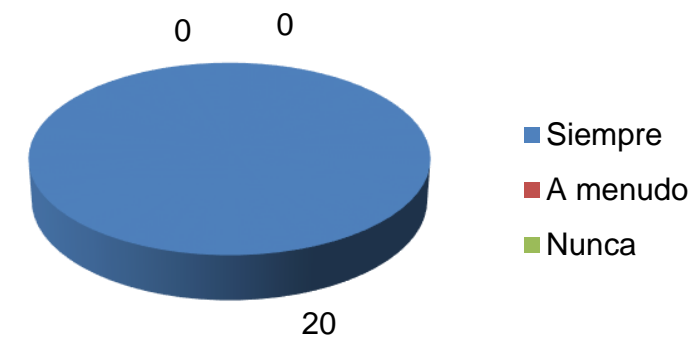

Figura 12. Influencia de la variación de precios en el nivel de consumo

\section{CONCLUSIONES}

El desahorro transitorio de algunas familias, es influenciado por los bajos ingresos y la presencia de un solo proveedor de ingresos en el hogar, esta situación plantea las desigualdades en las posibilidades de ahorro en los hogares de los sectores urbanos frente a los rurales, con las condiciones de demanda de mercado laboral (9). El estudio sobre la influencia de la equidad de género en las decisiones de consumo y ahorro familiares de la parroquia El Retiro, Cantón Machala, precisaron las siguientes conclusiones:

El criterio percibido a través de la entrevista al líder de la junta parroquial, permite enfatizar que aún el hombre sigue desempeñando como cabeza de hogar, el sector productivo motor de la parroquia es logrado a través de la producción bananera, camaronera con su aporte en la comunidad en lo laboral con la participación del hombre y mujer con igualdad de oportunidades en las funciones e ingresos. Es decir, la mejora de la economía local, ha sido por la actuación productiva de las familias en las diferentes faenas del campo y comercio. Actualmente el hombre y mujer trabajan y contribuyen a la economía familiar con equidad en la distribución de la renta.

Las encuestas dirigidas a los jefes (as) de hogar, fueron atendidas en su gran mayoría por hombres y un menor porcentaje de mujeres, ambos comentaron que existe participación equitativa en las decisiones económicas de las familias seleccionadas, las encuestas concluyeron interesantes aportes, como conocer que el jefe de hogar destaca al sexo masculino, el número de miembros o cargas familiares supera los 3, el ahorro equivale de 1 a $10 \%$ del promedio de la renta percibida, la mayoría destinada para futuras enfermedades $y$ educación, sin descartar que la dependencia de la actividad económica del cantón. Las situaciones para que la mujer permanezca en casa son por el cuidado de hogar y negocio, el nivel del estatus social es de clase baja y media.

Las bases teóricas establecen que la propensión marginal al consumo y al ahorro son positivas y menores a uno, en el caso del consumo es mayor que el ahorro, a medida que aumenta la renta aumenta el ahorro generando más oportunidades de gasto de consumo básicos y un efecto contrario cuando disminuye la renta; esta dinámica es similar a las actuaciones de las familias de la parroquia El Retiro, pues destinan un bajo porcentaje al ahorro ante un mayor consumo causado, corroborando que el nivel de renta afecta en las decisiones de gasto y reservas en las economías domésticas.

Se concluye que la eficiencia de las capacidades no está determinada por el género en cualquier aspecto o rol social, y los factores de distribución de compra y ahorro son decisiones que se deben tomar en función del nivel de la renta percibida y de los principales miembros de la unidad familiar hombre y mujer, a pesar de las diferencias sociales en el sector rural y urbano.

\section{REFERENCIAS}

1. Universidad de Valencia. Educación para la igualdad de género: Un modelo de evaluación. [Internet]. Valencia. [Consultado 23 de septiembre 2016]. Disponible en: https://www.uv.es/lisis/amapola/2015/educacion _igualdad.pdf. (2015).

2. The EU-LAC Innovation Portal Americas. Igualdad y Equidad de Género: un panorama internacional. [Internet]. [Consultado 23 de septiembre 2016]. Disponible en: http://www.americasportal.eu/sites/americasport al.eu/files/IsabelGenero.pdf. (2014).

3. Ruiz MT, Guijarro M, Rodenas C, Martí M, Jiménez MD, Fernández J. Tiempos de equidad de género: descripción de las desigualdades entre comunidades autónomas, España 20062014. Gaceta Sanitaria. [Revista en internet]. [Consultado 23 de septiembre 2016]. 30(4): p. 250-257 Disponible en: 
http://www.sciencedirect.com/science/article/pii/ S0213911116300450. (2016).

4. Cerva D. Participación política y violencia de género en México. Revista Mexicana de Ciencias Políticas y Sociales. [Revista en internet]. [Consultado 23 de septiembre 2016]. 15(222): p. 117-140 Disponible en: http://www.sciencedirect.com/science/article/pii/ S0185191814702120. (2014).

5. Lara G, Colín G. Sociedad de consumo y cultura consumista. Argumentos Scielo. [Revista en Internet] México. [Consultado 23 de septiembre 2016]. Disponible en: http://www.scielo.org.mx/scielo.php?script=sci_a rttext\&pid=S0187-57952007000300008 (2007).

6. Camacho F. Evolución del consumo en México. Aportes. May; VIII (ISSN 1665 1219): p. 75-88. (2003).

7. Carrasco A. La sociedad de consumo: Origen y Características. Contribuciones a la Economía. (2007).

8. Fondo de las Naciones Unidas para la Infancia (UNICEF). [Internet]. [Consultado 23 de septiembre 2016]. Disponible en: http://www.unicef.org/spanish/sowc07/profiles/in equality_household.php. (2006).

9. Armendáriz E. Micro finanzas familiares y los niveles de ahorro: Una visión Estratégica. Revista de la Universidad de Guayaquil. [Revista en Internet] p. 29-44 Disponible en: http://www.revistauniversidad.edu.ec/PDF/Edici on117.pdf. (2013).

10. Calderón J, Alcívar C. Comparación de los dos últimos años en los índices de políticas de ahorro y consumo en las familias del Ecuador. Observatorio de la Economía Latinoamericana. p. 1-16. (2014).

11. Instituto Nacional de Estadísticas y Censos (INEC). Encuesta nacional de Ingresos y Gastos de los hogares urbanos y rurales 2011-2012 [Internet]. [Consultado 31 de agosto 2016]. Disponible

en: http://www.ecuadorencifras.gob.ec/documentos/ web-

inec/Estadisticas_Sociales/Encuesta_Nac_Ingre sos_Gastos_Hogares_Urb_Rur_ENIGHU/ENIG HU-2011-2012/Metologia_ENIGHUR_20112012_rev.pdf. (2012).

12. Banco Atlas. El consumo, la renta y el ahorro [Internet]. [Consultado 31 de agosto 2016]. Disponible http://www.abc.com.py/articulos/el-consumo-larenta-y-el-ahorro-726601.html. (2003).

13. Bosch A. Macroeconomía. 4th ed. Nueva York: Antoni Bosch editor. (2005).

14. Castaño R, González H. Ideas económicas mínimas. 22nd ed. Bogotá: País Colombia: Ecoe Ediciones. (2011).

15. Spencer M. Economía contemporánea New York: Reverté S.A.(1993).

16. Biblioteca Virtual Eumed. Biblioteca Virtual de Derecho, Economía y Ciencias Sociales. [Internet]. [Consultado 31 de agosto 2016]. Disponible en: http://www.eumed.net/librosgratis/2007a/233/2m.htm._(2007)

17. Mankiw G. Principios de Economía México: Cengage Learning Editores. (2012).

18. Banco Central del Ecuador (BCE). Banco Central del Ecuador. [Internet]. [Consultado 31 de agosto 2016]. Disponible en: https://contenido.bce.fin.ec/documentos/Publica cionesNotas/Catalogo/NotasTecnicas/nota35.pd f_(1996).

19. Avila $H$. Introducción a la metodología de la investigación Chuhuahua, México: Eumed net; 2006.

20. Senplades. Plan Estrategico de Desarrollo de la Provincia de EI Oro. [Internet]. [Consultado 31 de agosto 2016]. Disponible en: http://www.eloro.gob.ec/wp-

content/uploads/2013/12/diagsocprov.pdf.(2013)

21. Senplades. Secretaría Nacional de Planificación y Desarrollo Cantón MACHALA, Provincia de EL ORO se encuentra en la Zona 7 de planificación. [Internet]. [Consultado 31 de agosto 2016]. Disponible en: http://app.sni.gob.ec/sni-

link/sni/Portal\%20SNI\%202014/FICHAS\%20F/0 701_MACHALA_EL\%20ORO.pdf (2014).

22. GAD Parroquial El Retiro. Gobierno Autónomo Descentralizado Parroquial El Retiro. [Internet]. Disponible en: www.elretiro.gob.ec. (2015). 